\title{
CD74-immunoreactive activated M1 microglia are shown late in the gerbil hippocampal CA1 region following transient cerebral ischemia
}

\author{
IN KOO HWANG ${ }^{1 *}$, JOON HA PARK ${ }^{2 *}$, TAE-KYEONG LEE ${ }^{3}$, DAE WON KIM $^{4}$, KI-YEON YOO $^{5}$, JI HYEON AHN ${ }^{2}$, \\ YANG HEE KIM ${ }^{6}$, JUN HWI CHO ${ }^{7}$, YOUNG-MYEONG KIM ${ }^{8}$, MOO-HO WON $^{3}$ and SEUNG MYUNG MOON ${ }^{9}$ \\ ${ }^{1}$ Department of Anatomy and Cell Biology, College of Veterinary Medicine, Research Institute for Veterinary Science, \\ Seoul National University, Seoul 08826; ${ }^{2}$ Department of Biomedical Science and Research Institute for Bioscience and \\ Biotechnology, Hallym University, Chuncheon, Gangwon 24252; ${ }^{3}$ Department of Neurobiology, School of Medicine, \\ Kangwon National University, Chuncheon, Gangwon 24341; Departments of ${ }^{4}$ Biochemistry and Molecular Biology \\ and ${ }^{5}$ Oral Anatomy, Research Institute of Oral Sciences, College of Dentistry, Gangneung-Wonju National University, \\ Gangneung, Gangwon 25457; Departments of ${ }^{6}$ Surgery, ${ }^{7}$ Emergency Medicine and ${ }^{8}$ Molecular and Cellular Biochemistry, \\ School of Medicine, Kangwon National University, Chuncheon, Gangwon 24341; ${ }^{9}$ Department of Neurosurgery, \\ Dongtan Sacred Heart Hospital, College of Medicine, Hallym University, Hwaseong, Gyeonggi 18450, Republic of Korea
}

Received July 14, 2016; Accepted March 8, 2017

DOI: $10.3892 / \mathrm{mmr} .2017 .6525$

\begin{abstract}
Activated M1 microglia secrete proinflammatory cytokines into damaged brain areas. The present study examined activated M1 microglial morphology and expression in the hippocampal Cornu Ammonis (CA) 1 region, which is vulnerable to transient ischemia. Transient cerebral ischemia was performed for $5 \mathrm{~min}$ in gerbils, and neuronal death in the CA1 region following transient cerebral ischemia was confirmed using cresyl violet staining, neuronal nuclear antigen immunohistochemistry and Fluoro-Jade B histofluorescent staining. In addition, CA1 regions were stained for cluster of differentiation (CD) 74, a marker for activated M1 microglia and a ligand for macrophage migration inhibitory factor In sham-operated animals, no CD74 immunoreactivity was observed in the hippocampal CA1 region. CD74
\end{abstract}

Correspondence to: Professor Moo-Ho Won, Department of Neurobiology, School of Medicine, Kangwon National University, 1 Gangwondaehak-gil, Chuncheon, Gangwon 24341, Republic of Korea

E-mail: mhwon@kangwon.ac.kr

Professor Seung Myung Moon, Department of Neurosurgery, Dongtan Sacred Heart Hospital, College of Medicine, Hallym University, 7 Keunjaebong-gil, Hwaseong, Gyeonggi 18450, Republic of Korea

E-mail: nsmsm@chol.com

*Contributed equally

Key words: transient ischemia, hippocampus, pyramidal neurons, neuronal death, gliosis, activated M1 microglia immunoreactivity was not observed in the hippocampal CA1 region until 3 days post-ischemic insult; however, elevated CD74 immunoreactivity was detected in the CA1 region from 5 days post-ischemia. Double immunofluorescence staining for CD74 and ionized calcium-binding adapter molecule 1, a marker for M1 microglial cells, confirmed the expression of CD74 on this microglial subtype. These results indicated that M1 microglia are activated late in the hippocampal CA1 region following ischemic stroke. Therefore, optimizing the timing of therapeutic intervention may reduce activated M1 microglial-induced neuronal damage.

\section{Introduction}

Acute ischemic brain damage results in severe neuronal loss via an inflammatory process, which predominantly involves invading leukocytes, activated resident microglia and macrophages $(1,2)$. Microglia form the first line of defense and control the immune response in the brain (3-5). It may be speculated that the microglia serve an essential role in forming a link between the central nervous system (CNS), a barrier-protected organ, and the general immune system; microglia are resident macrophages of the CNS and thus form an interface between the neural parenchyma and the immune system $(3,6)$. Little is known about the microglia in the healthy CNS; however, they are rapidly activated in acute pathological events, which may affect the CNS.

Activated microglia have dual effects. They may destroy invading microorganisms, remove potentially deleterious debris, promote tissue repair by secreting wound-healing factors and thus facilitate the return to tissue homeostasis $(7,8)$. However, these cells secrete large amounts of cytotoxic mediators such as reactive oxygen intermediates (9), nitric oxide (10), proteinases, excitatory amino acids (11) and 
inflammatory mediators, including interleukin-1 (IL-1) and IL-6 (12,13).

The activation of microglia and their histological localization is important in elucidating their roles following ischemic insults. Our previous study demonstrated the chronological alteration of ionized calcium-binding adapter molecule 1 (Iba-1), which is a marker for microglia, in the hippocampal Cornu Ammonis (CA) 1 region, induced by 5 min of transient forebrain ischemia using Mongolian gerbils (14). Previous research has demonstrated that microglia may be divided into activated M1 and M2 microglia, according to their phenotypes and polarization (15); activated M1 microglia secrete proinflammatory cytokines and are potentially harmful, whereas activated M2 microglia serve important roles in repair and plasticity (15-17). Improving our understanding of M1 microglia/macrophages is important because this subtype predominates within injured areas, and may accelerate neuronal damage via the release of inflammatory mediators, including tumor necrosis factor- $\alpha$ and nitric oxide (15). However, a recent study indicated that early-stage Alzheimer's patients exhibit M1-polarized microglia, whereas severe-stage plaques demonstrated an M2a-polarized phenotype (18). Notably, the concept of microglial M1 and M2 phenotypes has also been investigated in the field of stroke research (19). Cluster of differentiation (CD) 74 is a marker for activated M1 microglia because it serves as a chaperone for major histocompatibility complex class II (MHCII) molecules in antigen presenting cells, and as a receptor binding site for macrophage migration inhibitory factor (MIF). Numerous previous in vivo studies have focused on activated M1 microglia using animal models of focal ischemia. Therefore, the present study investigated the chronological alteration of activated microglia expressing CD74 in the hippocampus, induced by transient forebrain ischemia in Mongolian gerbils, which have been used as an animal model of transient forebrain ischemia (20-23).

\section{Materials and methods}

Induction of transient cerebral ischemia. A total of 35 male gerbils (age, 6 months; weight, 70-80 g) were obtained from Experimental Animal Center of Kangwon National University (Chuncheon, South Korea). Gerbils were housed in a conventional facility under stable temperature $\left(23^{\circ} \mathrm{C}\right)$ and humidity $(60 \%)$ with a $12-\mathrm{h}$ light/12-h dark cycle, and were provided with free access to food and water. The procedures for animal handling were in compliance with current international laws and policies (Guide for the Care and Use of Laboratory Animals, The National Academies Press, 8th Ed., 2011) (24), and were approved by the Institutional Animal Care and Use Committee (IACUC) at Kangwon National University (Chuncheon, South Korea; approval no. KW-130424-1).

Transient cerebral ischemia surgery was performed as previously described (25). In brief, animals were anesthetized with a mixture of $2.5 \%$ isoflurane (Ilsung Pharmaceuticals, Co., Ltd., Seoul, Korea) in 33\% oxygen and $67 \%$ nitrous oxide. The bilateral common carotid arteries were occluded for $5 \mathrm{~min}$, and the complete interruption of blood flow was confirmed by observation of the retinal central artery under an ophthalmoscope. A normothermic $\left(37 \pm 0.5^{\circ} \mathrm{C}\right)$ condition was maintained prior to, during and following surgery, until the animals had completely recovered from anesthesia. Sham-operated animals were subjected to the same surgical procedures; however, the common carotid arteries were not occluded.

Tissue processing. Tissues were collected as previously described (26). In brief, sham-operated ( $n=7)$ and ischemia-operated gerbils ( $\mathrm{n}=28$ in total; 7 gerbils/time point) were anesthetized with pentobarbital sodium $(40 \mathrm{mg} / \mathrm{kg}$; JW Pharmaceutical, Co., Ltd., Seoul, Korea) and perfused transcardially with $4 \%$ paraformaldehyde 1, 3, 5 and 7 days following reperfusion. Brain tissues were removed and serially cut into $30-\mu \mathrm{m}$ coronal sections.

Cresyl violet $(C V)$ staining. To examine cellular distribution and damage, $\mathrm{CV}$ staining was performed as previously described (26). In brief, CV acetate (Sigma-Aldrich; Merck KGaA, Darmstadt, Germany) was dissolved at a concentration of $1.0 \%(\mathrm{w} / \mathrm{v})$, and $0.28 \%$ glacial acetic acid was added. Sections were stained and subsequently dehydrated by immersing in serial ethanol baths.

Fluoro-Jade $B(F-J B)$ staining. To examine neuronal damage/death following transient ischemia, F-J B (a high affinity fluorescent marker for the localization of neuronal degeneration) histofluorescence staining was performed, according to a previously published procedure (27). Briefly, sections were first immersed in a solution containing $1 \%$ sodium hydroxide, transferred to a solution of $0.06 \%$ potassium permanganate, and subsequently transferred to a $0.0004 \%$ Fluoro-Jade B (Histo-chem, Inc., Jefferson, AR, USA) solution. After washing, the sections were placed on a slide warmer $\left(\sim 50^{\circ} \mathrm{C}\right)$, and examined using an epifluorescent microscope (Zeiss GmbH, Jena, Germany) with blue (450-490 nm) excitation light and a barrier filter. Digital images of the stained hippocampus were captured with an AxioM1 light microscope equipped with an Axiocam digital camera (both from Zeiss $\mathrm{GmbH})$, connected to a PC monitor.

Immunohistochemistry. Immunohistochemistry was performed out according to a previously published procedure (28). In brief, sections were incubated with mouse anti-neuronal nuclear antigen (NeuN; cat. no. MAB377; 1:1,000; EMD Millipore, Billerica, MA, USA), mouse anti-CD74 (cat. no. MCA46R; MHCII/RT1B clone; 1:100; Bio-Rad Laboratories, Inc., Hercules, CA, USA) or rabbit anti-Iba-1 (cat. no. 019-19741; 1:800; Wako Pure Chemical Industries, Ltd., Osaka, Japan) primary antibodies overnight at $4^{\circ} \mathrm{C}$, and subsequently incubated with biotinylated goat anti-mouse immunoglobulin (Ig)-G (cat. no. BA-9200; 1:200; Vector Laboratories, Inc., Burlingame, CA, USA) or goat anti-rabbit IgG (cat. no. BA-1000; 1:200; Vector Laboratories, Inc.) secondary antibodies for $2 \mathrm{~h}$ at room temperature, followed by incubation with a streptavidin-peroxidase complex (cat. no. SA-5004; 1:200; Vector Laboratories, Inc.) for $45 \mathrm{~min}$ at room temperature. Immunostaining was visualized with diaminobenzidene. Digital images of the stained tissue were captured with an AxioM1 light microscope equipped with an Axiocam digital camera, connected to a PC monitor. 
Double immunofluorescence. To examine the colocalization of CD74 and I1, the sections were processed by double immunofluorescence staining according to a previously published procedure (28). Briefly, sections were incubated with mouse anti-CD74 (cat. no. MCA46R; 1:25; Bio-Rad Laboratories, Inc.) and rabbit anti-Iba-1 (cat. no. 019-19741; 1:100; Wako Pure Chemical Industries, Ltd.) primary antibodies overnight at $4^{\circ} \mathrm{C}$, and subsequently incubated with $\mathrm{Cy} 3$-conjugated donkey anti-rabbit IgG (cat. no. 711-165-152; 1:200) and fluorescein isothiocyanate-conjugated donkey anti-mouse IgG (cat. no. 715-096-151; 1:200) secondary antibodies (both from Jackson ImmunoResearch Laboratories, Inc., West Grove, PA, USA) for $2 \mathrm{~h}$ at room temperature. Immunoreactions were observed under a LSM510 META NLO confocal microscope (Zeiss $\mathrm{GmbH})$.

Data analysis. Numbers of $\mathrm{NeuN}$-immunoreactive and F-J B-positive cells were analyzed according to a previously published method (26). In brief, cells were counted in a $250 \times 250 \mu \mathrm{m}$ square applied approximately at the center of the hippocampal CA1 region, using an image analyzing system (Optimas 6.5; CyberMetrics Corporation, Phoenic, AZ, USA). The studied tissue sections were selected at $120-\mu \mathrm{m}$ intervals, and cell counts were obtained by averaging the total cell numbers of 5 sections taken from each animal per group, between $1.4 \mathrm{~mm}$ and $2.0 \mathrm{~mm}$ posterior to the bregma, as defined by the gerbil atlas (29).

Densities of Iba-1 and CD74 immunoreactive structures were measured as previously described (26). Images were calibrated into an array of $512 \times 512$ pixels, corresponding to a tissue area of $250 \times 250 \mu \mathrm{m}$ (primary magnification, $\times 40$ ) and including the stratum pyramidale. The densities of all Iba-1 and CD74 immunoreactive structures were evaluated on the basis of their optical density (OD), which was obtained following transformation of the mean gray level using the formula: $\mathrm{OD}=\log (256 /$ mean gray level). The background OD was determined from areas adjacent to the measured area. After the background density was subtracted, a ratio of the image OD was calibrated as \% relative OD (ROD), using Adobe Photoshop version 8.0 (Adobe Systems, Inc., Beijing, China) and analyzed using ImageJ version 1.50 software (National Institutes of Health, Bethesda, MD, USA).

Statistical analysis. Data are presented as the mean \pm standard. Differences between the means were statistically analyzed by one-way analysis of variance followed by a Bonferroni's post-hoc test, using GraphPad Prism 5.01 software (GraphPad Software, Inc., La Jolla, CA, USA). P<0.05 was considered to indicate a statistically significant difference.

\section{Results}

Neuronal damageldeath. CV, NeuN and F-J B staining was conducted in the CA1 hippocampal region following transient ischemia in gerbils (Fig. 1). In the sham-operated group, CV-positive cells and NeuN-immunoreactive neurons were clearly observed in the stratum pyramidale of the hippocampal CA1 region (Fig. 1A, a, and D). In contrast, no F-J B positive cells were detected in the hippocampal CA1 region of these gerbils (Fig. $1 \mathrm{G}$ and $\mathrm{J}$ ). The 1 day after ischemic insult, the distribution pattern of CV-positive cells and $\mathrm{NeuN}$-immunoreactive neurons in the $\mathrm{CA} 1$ region was similar to that in the sham-operated group (Fig. 1B, b and E), and no F-J B positive cells were observed in the hippocampal $\mathrm{CA} 1$ region in this group (Fig. $1 \mathrm{H}$ and $\mathrm{J}$ ). $\mathrm{CV}$-positive cells were significantly decreased in the stratum pyramidale of the CA1 region 5 days after ischemic insult (Fig. 1C and c). Similarly, only a few NeuN immunoreactive neurons were detected in the stratum pyramidale of the CA1 region (Fig. $1 \mathrm{~F}$ and $\mathrm{J}$ ), however, many F-J B positive cells were observed in the stratum pyramidale of the CA1 region 5 days after ischemic insult (Fig. 1I and J). These results indicated that transient cerebral ischemia-induced neuronal death in pyramidal neurons of the hippocampal CA1 region occurred 5 days after ischemic insult.

Iba-1 immunoreactive microglia. Iba-1 and CD74 immunoreactivity was detected in the hippocampal CA1 region following transient ischemia in gerbils (Fig. 2). In the sham-operated group, Iba-1-immunoreactive microglia, in a resting form, were distributed in all layers of the CA1 region and had a typical ramified morphology with small areas of cytoplasm (Fig. 2A). The day after ischemic insult, Iba-1-immunoreactive microglia demonstrated activation; the cell bodies became hypertrophied with thickened processes (Fig. 2B) and their ROD was increased compared with the sham-operated group. A total of 3 days after ischemic insult, the majority of Iba-1-immunoreactive microglia were more hypertrophied in shape and the ROD of these cells was significantly increased, compared with those at 1 day post-ischemia (Fig. 2C and $\mathrm{K}$ ). After 5 days, Iba-1-immunoreactive microglia were aggregated in the stratum pyramidale of the CA1 region (Fig. 2D). After 7 days, the aggregation of Iba-1-immunoreactive microglia, and their ROD, was significantly increased, compared with 5 day post-ischemic tissues (Fig. 2E and K).

CD74-immunoreactive microglia. In the sham-operated group, CD74 immunoreactivity was not detected in any cells in the CA1 region (Fig. 2F). Between days 1 and 3 after ischemic insult, very few CD74-immunoreactive cells were observed in the CA1 region (Fig. 2G and $\mathrm{H}$ ); however, CD74-immunoreactive cells were detected in the strata oriens and radiatum of the CA1 region 5 days after ischemic insult (Fig. 2I and K). After 7 days, the number of CD74-immunoreactive cells were further elevated in the CA1 region, compared with those at 5 days post-ischemia (Fig. $2 \mathrm{~J}$ and $\mathrm{K}$ ).

Co-localization of Iba-1 and CD74. Double immunofluorescence staining indicated that Iba-1 immunoreactivity was observed in the CA1 region of the sham-operated group; however, no CD74 immunoreactivity was observed in this group (Fig. 3A-C). After 7 days, CD74 immunoreactivity was observed in numerous cells in the CA1 region, and this appeared to colocalize with Iba-1 in the microglia (Fig. 3D-G).

\section{Discussion}

Microglial reactivity in the ischemic brain is closely associated with the development of delayed neuronal cell death in 


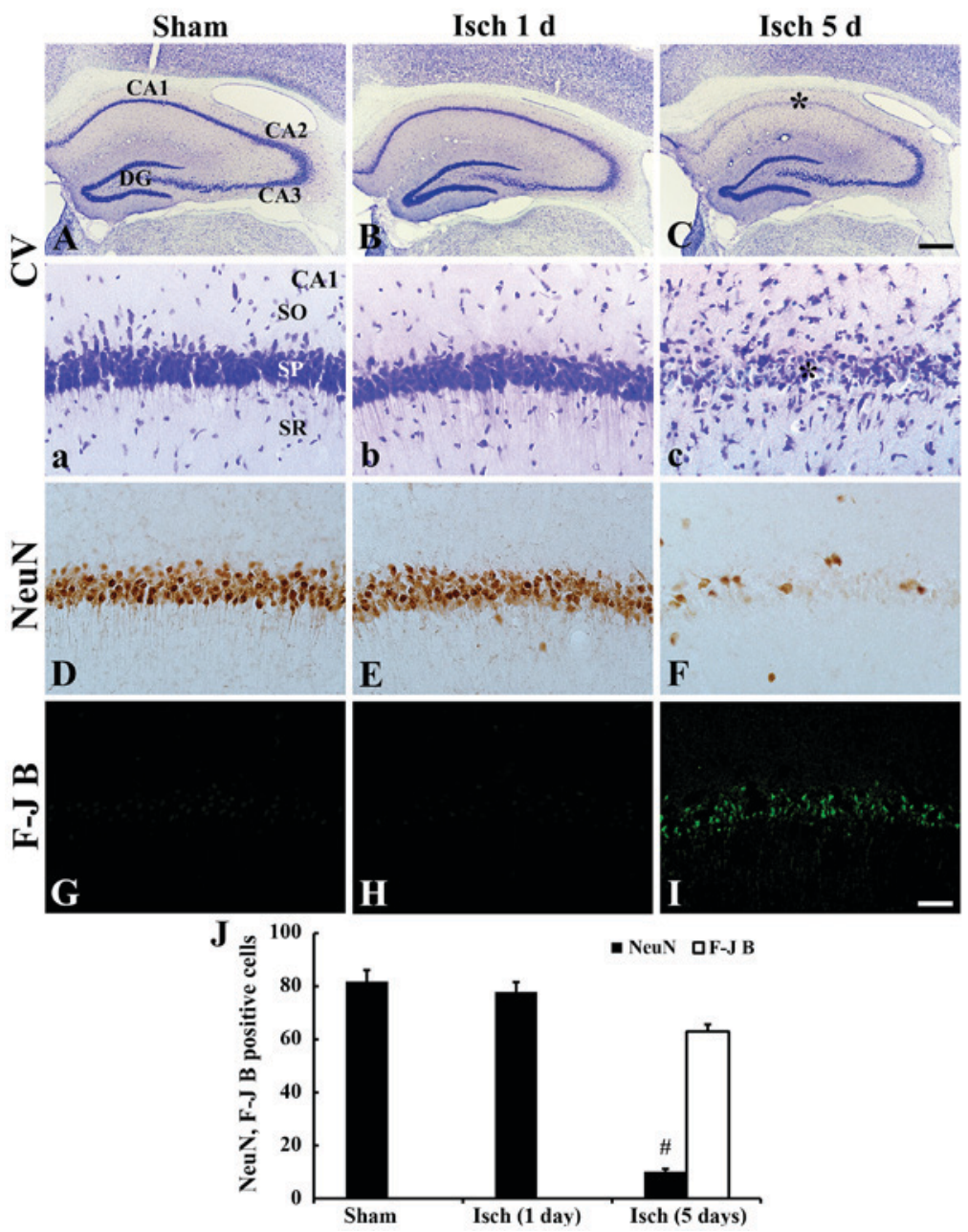

Figure 1. CV, NeuN and F-J B staining in the CA1 hippocampal region following transient ischemia in gerbils. (A-C, a-c) CV, (D-F) NeuN and (G-I) F-J B staining. Staining of the hippocampal CA1 region of (A, a, D, G) sham-operated and (B, C, b, c, E, F, H, I) ischemia-operated gerbils was performed. CV-and NeuN-positive cells were clearly observed in the sham-operated group; however, F-J B positive cells were not detected. A few CV and NeuN immunoreactive cells were present in the SP (") of the CA1 region 5 days after ischemic insult, and several F-J B positive cells were present in the SP at this time point. Scale bar $=50 \mu \mathrm{m}$. (J) Mean number of NeuN or F-J B positive cells in the CA1 region, $\mathrm{n}=7 /$ group. Data are presented as the mean \pm standard error. ${ }^{\#} \mathrm{P}<0.05$, vs. sham. CA, Cornu Ammonis; CV, cresyl violet; F-J B, fluoro-Jade B; NeuN, neuronal nuclear antigen; SP, stratum pyramidale; SO, stratum oriens; SR, stratum radiatum; Isch, ischemia; DG, dentate gyrus.

vulnerable regions $(4,30)$. An early and transient microglial reaction occurs throughout the majority of the hippocampus within $24 \mathrm{~h}$ of ischemia, including the hippocampal CA3 region, where no subsequent neuronal loss occurs (31). In addition, our previous study demonstrated chronological alterations in Iba-1-positive microglia in the hippocampus after 5 min of transient cerebral ischemia in gerbils (14). However, to the best of our knowledge, few studies have investigated the timing of microglia activation in the hippocampus, following induction by transient cerebral ischemia.

The Iba-1 gene is located within the MHC class III region of the brain (32), and is specifically expressed in microglia $(14,32,33)$. Microglia proliferate within selectively vulnerable brain areas with ischemia-induced neuronal cell death during the first $48 \mathrm{~h}$ after ischemic injury $(34,35)$. Differential analysis of the M1 and M2 subtypes is important, because M1 and M2 microglia demonstrate contradictory functions in the inflammatory process of neurological disorders. The present study observed a chronological change in M1 polarization (MHC-II- and CD74-positive cells) in the hippocampal CA1 region after 5 min of transient cerebral ischemia in gerbils.
These results will develop our understanding of the cell death process in the hippocampus, following ischemic insults.

CD74 immunoreactivity was not observed in the hippocampus of the sham-operated gerbils; however, CD74 was expressed in Iba-1-immunoreactive microglia in the hippocampal CA1 region, $\geq 5$ days after ischemic insult. In the hippocampal CA1 region, delayed neuronal death occurs several days after transient cerebral ischemia in rodents (36-38). In this regard, the present study observed M1 polarization after neuronal death of CA1 pyramidal neurons. In particular, CD74 immunoreactivity gradually increased in the hippocampal CA1 region $\geq 5$ days following ischemic insult. Notably, in a rat model of focal cerebral ischemia induced by middle cerebral artery occlusion, expression of the M1 marker CD16/32 was significantly increased 3 days after ischemic insult and remained elevated 14 days after focal ischemia (16). Furthermore, the authors reported that other M1-type genes, including inducible nitric oxide synthase and CD11b, also gradually increased 3 days after ischemia and remained at high levels 14 days later (16).

CD74 is also a ligand for MIF, which is released by a variety of cell types. MIF is a proinflammatory cytokine that 


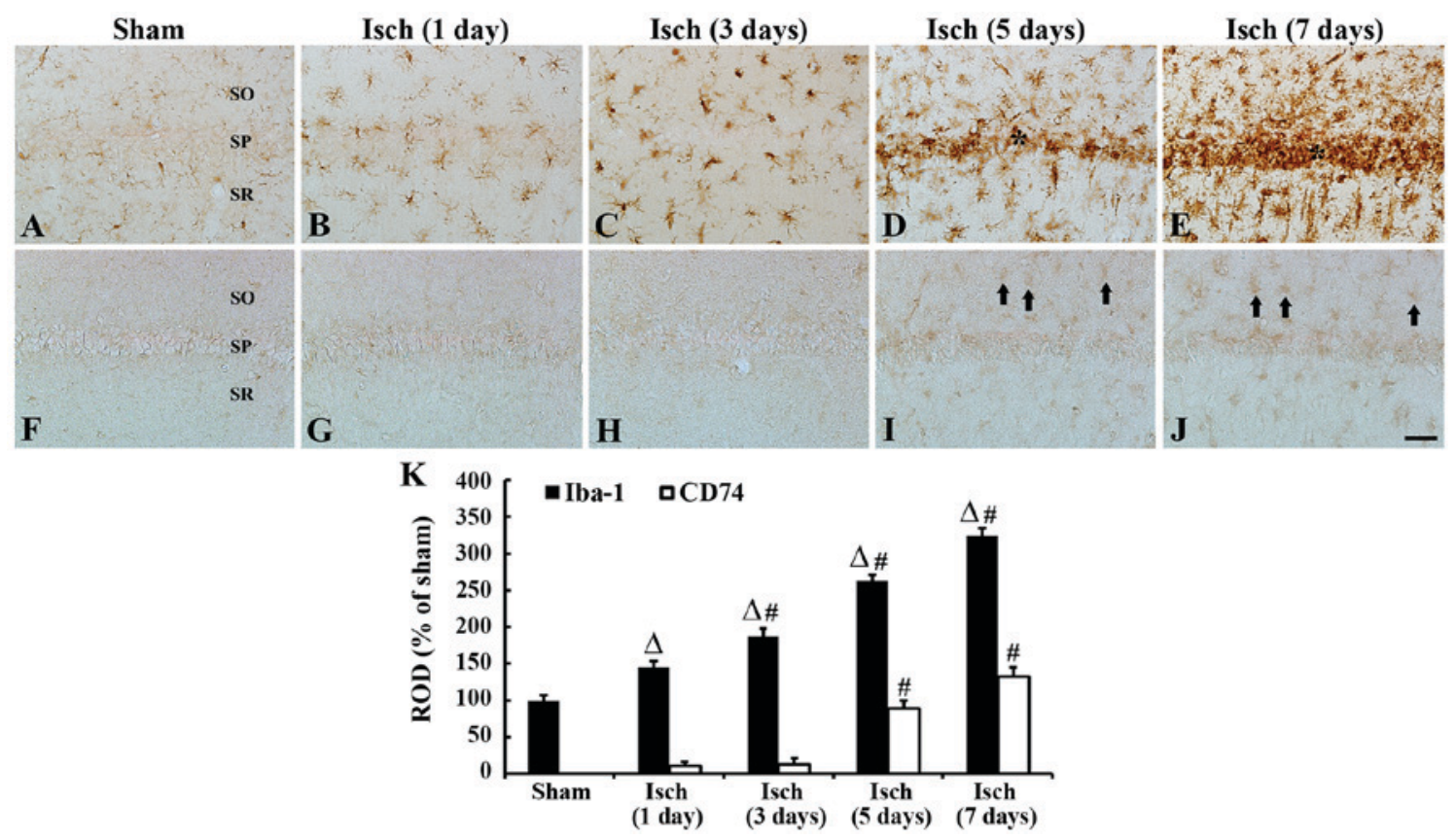

Figure 2. Iba-1 and CD74 immunoreactivity in the hippocampal CA1 region, following transient ischemia in gerbils. Immunohistochemical staining of (A-E) Iba-1 and (F-J) CD74. Staining was performed in (A and F) sham-operated and (B-E and G-J) ischemia-operated gerbils. In the sham-operated group, only Iba-1 immunoreactive microglia were observed. After 5-7 days, numerous Iba-1 immunoreactive microglia became aggregated in the SP ("), and CD74-immunoreactive cells (black arrows) were scattered in the CA1 region. Scale bar $=50 \mu \mathrm{m}$. (K) ROD as percentage of Iba-1 and CD74 immunoreactive structures in the CA1 region of the sham-and ischemia-operated groups, $\mathrm{n}=7 /$ group. ${ }^{\Delta} \mathrm{P}<0.05$ vs. sham; ${ }^{*} \mathrm{P}<0.05$, vs. the respective pre-time point group. Data are presented as the mean \pm standard error. Iba-1, ionized calcium-binding adapter molecule 1; CD, cluster of differentiation; CA, Cornu Ammonis; SP, stratum pyramidale; SO, stratum oriens; SR, stratum radiatum; Isch, ischemia; ROD, relative optical density; Isch, ischemia.
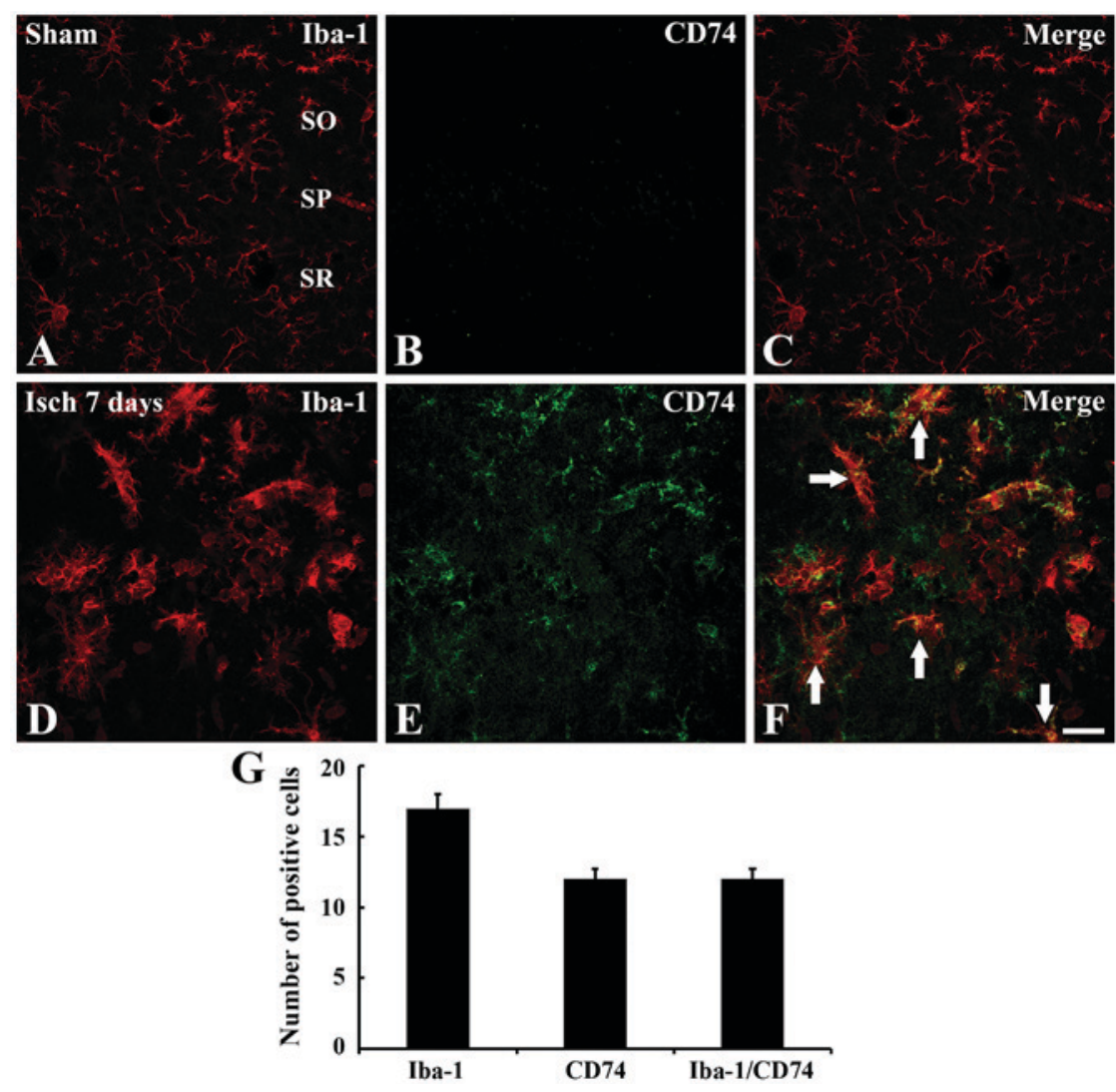

Figure 3. Double immunofluorescence staining for Iba-1 and CD74 in the hippocampal CA1 region. Immunofluorescence staining in the sham group for (A) Iba-1, (B) CD74 and (C) their merged overlay. Immunfluorescence staining in the ischemic model group for (D) Iba-1, (E) CD74 and (F) their merged overlay. White arrows indicate regions of Iba-1/CD74 overlap. Many of Iba-1 immunoreactive microglia demonstrate CD74 immunoreactivity in the CA1 region. Scale bar=50 $\mu \mathrm{m}$. (G) Mean number of Iba-1, CD74 and Iba-1/CD74-immunoreactive cells in the CA1 region 7 days after ischemia-reperfusion, $\mathrm{n}=7$ /group. Data are presented as the mean \pm standard error. Iba-1, ionized calcium-binding adapter molecule 1; CD, cluster of differentiation; CA, Cornu Ammonis; SO, stratum oriens; SP, stratum pyramidale; SR, stratum radiatum; Isch, ischemia. 
binds to CD74 and sequentially activates the extracellular signal-regulated kinase 1/2-dependent signal transduction pathway, cell proliferation and prostaglandin E2 production (39). Overexpression of MIF significantly decreases $\mathrm{H}_{2} \mathrm{O}_{2}$-induced cell death, and knockdown of the MIF gene exacerbates neuronal damage in an animal model of ischemic insult (23). The present study observed an increase of CD74 immunoreactivity in the gerbil hippocampal CA1 region; it may be hypothesized that this increase could protect neurons from ischemic damage. However, it was previously reported that MIF expression is decreased in the infarct area of the mouse brain following ischemia (23). Based on these findings, the expression pattern of CD74 may be varied in the damaged brain tissue according to ischemic insults, with differences based on whether the ischemia was global or focal, and the duration of the transient ischemic insult.

In conclusion, CD74-immunoreactive activated M1 microglia were observed a few days after transient cerebral ischemia in the gerbil hippocampal CA1 region. This observation indicates that activated M1 microglia may be closely associated with neuronal death in various microenvironments in the hippocampal CA1 region following transient forebrain ischemia. Therefore, it may be hypothesized that activated M1 microglia has potential as an alternative target for the development of novel therapeutic strategies for the treatment of patients with cerebral ischemia.

\section{Acknowledgements}

This work was supported by the Basic Science Research Program through the National Research Foundation of Korea (NRF) funded by the Ministry of Science, ICT and future Planning (MSIP; grant no. NRF-2014R1A2A2A01005307), and by the Bio \& Medical Technology Development Program of the NRF, funded by the Korean government, MSIP (grant no. NRF-2015M3A9B6066835).

\section{References}

1. Danton GH and Dietrich WD: Inflammatory mechanisms after ischemia and stroke. J Neuropathol Exp Neurol 62: 127-136, 2003.

2. Stoll G and Jander S: The role of microglia and macrophages in the pathophysiology of the CNS. Prog Neurobiol 58: 233-247, 1999.

3. Kreutzberg GW: Microglia: A sensor for pathological events in the CNS. Trends Neurosci 19: 312-318, 1996.

4. Lees GJ: The possible contribution of microglia and macrophages to delayed neuronal death after ischemia. J Neurol Sci 114 119-122, 1993.

5. Stoll G, Jander S and Schroeter M: Inflammation and glial responses in ischemic brain lesions. Prog Neurobiol 56: 149-171, 1998.

6. Kato $\mathrm{H}$ : The role of microglia in ischemic brain injury. In Inflammation and Stroke. Springer, pp89-pp99, 2001.

7. Nakajima K, Tsuzaki N, Shimojo M, Hamanoue M and Kohsaka S: Microglia isolated from rat brain secrete a urokinase-type plasminogen activator. Brain Res 577: 285-292, 1992.

8. Vaca K and Wendt E: Divergent effects of astroglial and microglial secretions on neuron growth and survival. Exp Neurol 118: 62-72, 1992.

9. Colton CA and Gilbert DL: Production of superoxide anions by a CNS macrophage, the microglia. FEBS Lett 223: 284-288, 1987.

10. Han HS, Qiao Y, Karabiyikoglu M, Giffard RG and Yenari MA Influence of mild hypothermia on inducible nitric oxide synthase expression and reactive nitrogen production in experimental stroke and inflammation. J Neurosci 22: 3921-3928, 2002.
11. Stumm R, Culmsee C, Schafer MK, Krieglstein J and Weihe E: Adaptive plasticity in tachykinin and tachykinin receptor expression after focal cerebral ischemia is differentially linked to gabaergic and glutamatergic cerebrocortical circuits and cerebrovenular endothelium. J Neurosci 21: 798-811, 2001.

12. Maeda Y, Matsumoto M, Hori O, Kuwabara K, Ogawa S, Yan SD, Ohtsuki T, Kinoshita T, Kamada T and Stern DM: Hypoxia/reoxygenation-mediated induction of astrocyte interleukin 6: A paracrine mechanism potentially enhancing neuron survival. J Exp Med 180: 2297-2308, 1994.

13. Suzuki S, Tanaka K, Nogawa S, Nagata E, Ito D, Dembo T and Fukuuchi Y: Temporal profile and cellular localization of interleukin-6 protein after focal cerebral ischemia in rats. J Cereb Blood Flow Metab 19: 1256-1262, 1999.

14. Hwang IK, Yoo KY, Kim DW, Choi SY, Kang TC, Kim YS and Won MH: Ionized calcium-binding adapter molecule 1 immunoreactive cells change in the gerbil hippocampal CA1 region after ischemia/reperfusion. Neurochem Res 31: 957-965, 2006.

15. Kigerl KA, Gensel JC, Ankeny DP, Alexander JK, Donnelly DJ and Popovich PG: Identification of two distinct macrophage subsets with divergent effects causing either neurotoxicity or regeneration in the injured mouse spinal cord. J Neurosci 29: 13435-13444, 2009.

16. Hu X, Li P, Guo Y, Wang H, Leak RK, Chen S, Gao Y and Chen J: Microglia/macrophage polarization dynamics reveal novel mechanism of injury expansion after focal cerebral ischemia. Stroke 43: 3063-3070, 2012.

17. Perego C, Fumagalli S and De Simoni MG: Temporal pattern of expression and colocalization of microglia/macrophage phenotype markers following brain ischemic injury in mice. J Neuroinflammation 8: 174, 2011.

18. Sudduth TL, Schmitt FA, Nelson PT and Wilcock DM: Neuroinflammatory phenotype in early Alzheimer's disease. Neurobiol Aging 34: 1051-1059, 2013.

19. Frieler RA, Meng H, Duan SZ, Berger S, Schütz G, He Y, Xi G, Wang MM amd Mortensen RM: Myeloid-specific deletion of the mineralocorticoid receptor reduces infarct volume and alters inflammation during cerebral ischemia. Stroke 42: 179-185, 2011.

20. Liu YR, Lei RY, Wang CE, Zhang BA, Lu H, Zhu HC and Zhang GB: Effects of catalpol on ATPase and amino acids in gerbils with cerebral ischemia/reperfusion injury. Neurol Sci 35: 1229-1233, 2014.

21. Min D, Mao X, Wu K, Cao Y, Guo F, Zhu S, Xie N, Wang L, Chen T, Shaw C and Cai J: Donepezil attenuates hippocampal neuronal damage and cognitive deficits after global cerebral ischemia in gerbils. Neurosci Lett 510: 29-33, 2012.

22. Qi J, Li Y, Zhang H, Cheng Y, Sung Y, Cao J, Zhao Y and Wang F: A novel conjugate of low-molecular-weight heparin and $\mathrm{Cu}, \mathrm{Zn}$-superoxide dismutase: Study on its mechanism in preventing brain reperfusion injury after ischemia in gerbils. Brain Res 1260: 76-83, 2009.

23. Zhang YB, Kan MY, Yang ZH, Ding WL, Yi J, Chen HZ and $\mathrm{Lu} Y$ : Neuroprotective effects of $\mathrm{N}$-stearoyltyrosine on transient global cerebral ischemia in gerbils. Brain Res 1287: 146-156, 2009.

24. Institute of Laboratory Animal Research, Committee for the Update of the Guide for the Care and Use of Laboratory Animals, National Research Council: Guide for the care and use of laboratory animals. 8th edition. National Academies Press, Washington, DC, p220, 2011

25. Lee CH, Park JH, Yoo KY, Choi JH, Hwang IK, Ryu PD, Kim DH, Kwon YG, Kim YM and Won MH: Pre- and post-treatments with escitalopram protect against experimental ischemic neuronal damage via regulation of BDNF expression and oxidative stress. Exp Neurol 229: 450-459, 2011.

26. Park JH, Shin BN, Chen BH, Kim IH, Ahn JH, Cho JH, Tae HJ, Lee JC, Lee CH, Kim YM, et al: Neuroprotection and reduced gliosis by atomoxetine pretreatment in a gerbil model of transient cerebral ischemia. J Neurol Sci 359: 373-380, 2015.

27. Schmued LC and Hopkins KJ: Fluoro-Jade B: A high affinity fluorescent marker for the localization of neuronal degeneration. Brain Res 874: 123-130, 2000.

28. Lee CH, Park JH, Cho JH, Ahn JH, Yan BC, Lee JC, Shin MC, Cheon SH, Cho YS, Cho JH, et al: Changes and expressions of Redd1 in neurons and glial cells in the gerbil hippocampus proper following transient global cerebral ischemia. J Neurol Sci 344: 43-50, 2014.

29. Loskota WJ, Lomax P and Verity MA: A stereotaxic atlas of the mongolian gerbil brain (Meriones unguiculatus). Mich Ann Arbor Science, 1974. 
30. Yan BC, Park JH, Ahn JH, Choi JH, Yoo KY, Lee CH, Cho JH, Kim SK, Lee YL, Shin HC and Won MH: Comparison of glial activation in the hippocampal CA1 region between the young and adult gerbils after transient cerebral ischemia. Cell Mo Neurobiol 32: 1127-1138, 2012.

31. Finsen BR, Jørgensen MB, Diemer NH and Zimmer J: Microglial MHC antigen expression after ischemic and kainic acid lesions of the adult rat hippocampus. Glia 7: 41-49, 1993.

32. Imai Y, Ibata I, Ito D, Ohsawa K and Kohsaka S: A novel gene iba1 in the major histocompatibility complex class III region encoding an EF hand protein expressed in a monocytic lineage. Biochem Biophys Res Commun 224: 855-862, 1996.

33. Ito D, Imai Y, Ohsawa K, Nakajima K, Fukuuchi Y and Kohsaka S: Microglia-specific localisation of a novel calcium binding protein, Iba1. Brain Res Mol Brain Res 57: 1-9, 1998.

34. Gehrmann J, Bonnekoh P, Miyazawa T, Hossmann KA and Kreutzberg GW: Immunocytochemical study of an early microglial activation in ischemia. J Cereb Blood Flow Metab 12: 257-269, 1992.
35. Gehrmann J, Bonnekoh P, Miyazawa T, Oschlies U, Dux E, Hossmann KA and Kreutzberg GW: The microglial reaction in the rat hippocampus following global ischemia: Immuno-electron microscopy. Acta Neuropathol 84: 588-595, 1992.

36. Kirino T: Delayed neuronal death in the gerbil hippocampus following ischemia. Brain Res 239: 57-69, 1982.

37. Miyawaki S, Imai H, Hayasaka T, Masaki N, Ono H, Ochi T, Ito A, Nakatomi H, Setou $\mathrm{M}$ and Saito N: Imaging mass spectrometry detects dynamic changes of phosphatidylcholine in rat hippocampal CA1 after transient global ischemia. Neuroscience 322: 66-77, 2016.

38. Pulsinelli WA, Brierley JB and Plum F: Temporal profile of neuronal damage in a model of transient forebrain ischemia. Ann Neurol 11: 491-498, 1982.

39. Leng L, Metz CN, Fang Y, Xu J, Donnelly S, Baugh J, Delohery T, Chen Y, Mitchell RA and Bucala R: MIF signal transduction initiated by binding to CD74. J Exp Med 197: 1467-1476, 2003 